\title{
Distance perception in virtual reality: A meta-analysis of the effect of head-mounted display characteristics
}

\author{
Jonathan W. Kelly
}

\begin{abstract}
Distances are commonly underperceived in virtual reality (VR), and this finding has been documented repeatedly over more than two decades of research. Yet, there is evidence that perceived distance is more accurate in modern compared to older head-mounted displays (HMDs). This meta-analysis, based on 137 data points from 61 publications, describes egocentric distance perception across $20 \mathrm{HMDs}$ and examines the relationship between perceived distance and technical HMD characteristics. Judged distance was positively associated with HMD field of view (FOV), positively associated with HMD resolution, and negatively associated with HMD weight. The effects of FOV and resolution were more pronounced among heavier HMDs. These findings suggest that future improvements in these technical characteristics may be central to resolving the problem of distance underperception in VR.
\end{abstract}

Index Terms-Distance perception, Egocentric distance, Virtual reality, Virtual environment, Meta-analysis, Head-mounted display, Resolution, Weight, Field of view.

\section{INTRODUCTION}

$\mathrm{V}$ IRTUAL reality (VR) provides the experience of a threedimensional virtual environment (VE) by displaying stereo 3D graphics that create optical stimulation similar to what is experienced when viewing a real environment. However, perceived spatial properties of a VE commonly differ from those of real environments. Research on this topic has focused heavily on perception of absolute egocentric distance (i.e., the absolute distance from the viewer to another location in the environment), although perception of other spatial properties has been evaluated through several measures including judgments of relative distance [1], [2], [3], room size [4], [5], [6], walking speed [7], and affordances [8], [9].

Distance underperception in VR has been documented repeatedly over the past two decades, beginning in the 1990s [10], [11], [12]. Most studies demonstrating underperception in VR have tested egocentric distances within "action space" [13], which constitutes the range of distances from arm's length to 30 meters. Results from studies on "personal space" within reaching distance are inconsistent in whether they indicate underperception or overperception [14], [15], [16], [17], [18], [19], [20], [21]. Although the problem of distance underperception within action space is persistent, there are two lines of evidence that the problem may have lessened in recent years. First, direct comparisons indicate that distance judgments are more accurate in newer headmounted displays (HMDs) compared to older ones [22], [23], [24], which suggests an improving trend. Second, there are reports of accurate distance judgments in modern HMDs, including studies reporting comparable distance judgments in real and virtual environments (HTC Vive: [24];

- J. W. Kelly is with the Department of Psychology, Iowa State Universty, Ames, IA, 50011.

E-mail: jonkelly@iastate.edu

Manuscript received XXXX XX, 2022; revised XXXXX XX 2022.
Oculus DK2: [25], Oculus CV1: [26]), as well as studies lacking a real environment comparison but reporting distance judgments quite close to $100 \%$ of the intended distance (HTC Vive: [27]; Oculus DK1: [22], [28]; Pimax 5k Plus: [29]). Examples such as these are notably absent from the literature using older HMDs. Yet, reports of accurate distance judgments in modern HMDs contrast with seemingly similar studies using many of the same modern HMDs but reporting at least some degree of distance underperception (Oculus DK2: [6], [22], [30], HTC Vive: [22], [31], [32], [33], Oculus CV1: [22], [33]).

This project reports a meta-analysis of the literature on distance perception in HMD-based VR. The first goal of this research was to more accurately estimate perceived distance in VR, both as an overall estimate across all studies and separately within each of several HMDs that have been commonly used in this research. Heterogeneity among studies using the same HMD is expected and is likely due to differences in experimental procedures and stimuli (e.g., VE characteristics), as well as sampling error. As an example of the heterogeneity in results across studies, reports of judged distance using the HTC Vive HMD have ranged from around $66 \%$ of intended distance [22] to $102 \%$ of intended distance [27]. Meta-analyzing several studies using the same HMD provides a good estimate of perceived distance for that specific HMD.

The second goal of the meta-analysis was to identify whether perceived distance is related to technical characteristics of the HMD, such as field of view (FOV), weight, and resolution. Many HMDs have been used over the past 20+ years of research on the topic, and comparison across studies using different HMDs may reveal whether some characteristics are especially predictive of distance perception accuracy. It is encouraging that distance perception appears more accurate in modern HMDs compared to older ones, but it would be valuable to understand potential causes 
of any such improvement. Meta-analysis of the literature on distance perception in VR could reveal some of these associations. The knowledge gained from this meta-analysis would inform theories about distance perception in VR, and might provide a road map for future technological improvements that will benefit distance perception in VR.

\subsection{HMD technical characteristics and their impact on perceived distance}

HMD technology has advanced considerably in the 20+ years since the first reports of distance underperception. Early HMDs produced in the 1990s were typically heavy, low resolution, and provided limited FOV. HMDs in the 2000s typically offered improved resolution and occasionally wider FOV, but weight typically remained high. The era of consumer-oriented HMDs began in the 2010s, first with companies like Oculus and HTC releasing development kits and eventually consumer products. These HMDs were lighter and had wider FOV, although resolution improvements did not arrive until later in the decade. Other HMD characteristics have certainly changed over time, such as display brightness and contrast, optics (e.g., the type of lens used), and ergonomics such as weight distribution on the head and head strap comfort. Weight, resolution, and FOV are the most easily quantified and most commonly reported characteristics of HMDs. HMD weight, FOV, and resolution have also been experimentally investigated as potential causes of distance underperception [22], [29], [34], [35], [36]. Thus, these factors represent the focus of this review and meta-analysis. This section reviews prior experimental research on the effects of HMD FOV, resolution, and weight on distance perception in VR.

Several studies have investigated the potential impact of FOV on perceived egocentric distance. Limited FOV might exclude relevant cues to egocentric distance, including texture information that specifies the ground plane. One type of experiment has approached this topic by comparing real world distance perception with full versus limited FOV. The results show that limiting real world FOV does not affect distance judgments when head rotations are allowed [35], but causes slight underperception when head rotations are restricted [36].

Another research approach is to manipulate the FOV experienced within the HMD. The earliest of these investigations used a boom-mounted (not head-mounted) display, whereby the display weight was carried by a boom capable of positioning the display in front of the viewer's eyes. Paradoxically, reduced FOV led to over-perception of distance, whereas the larger native FOV produced underperception [10]. A similar approach using wide FOV HMDs indicates that underperception is exaggerated by dramatic FOV reduction to around 60 degrees diagonal, compared to native FOV ranging from 110-200 degrees diagonal [22], [29], [37]. However, reduction from 200 to 110 degrees in one study did not affect judged distance [29], suggesting that there is a threshold below which distance is underperceived. Whereas most studies manipulated diagonal FOV, one study [38] independently manipulated vertical and horizontal FOV and found that only the horizontal FOV manipulation affected perceived distance.
HMD resolution has received limited research attention, perhaps because it is not easily manipulated in a controlled experiment, and because there is no equivalent manipulation in the real world. Before reviewing the literature, it is important to define resolution. Most consumers are familiar with resolution reported in the number of pixels, as is common with monitors, TVs, and phones. However, the image quality of a screen is affected by the number of pixels as well as the viewing distance, which collectively determine the density of the pixels projected on the viewer's eye (e.g., the number of pixels per degree of visual angle). Greater pixel density allows for richly detailed images, whereas lower pixel density creates chunky images and an experience in VR referred to as the screen door effect, where the pixelated scene looks as though it is being viewed through a screen. Low pixel density might therefore inhibit the perception of texture cues that are important for perceiving surface orientations.

A few studies have explored the effect of image resolution on perceived distance. Higher resolution images have been found to produce somewhat more accurate perception of depth when presented on a monitor, but not when presented in an HMD [34]. In another study, higher resolution images displayed on a projection screen led to more accurate distance judgments [39], but the sample size (4 participants) was minuscule. An augmented reality study presented images on a handheld screen and reported no effect of image resolution on perceived distance [40].

Other studies have evaluated the effect of image blur, whereby the scene in front and behind a specific distance is blurred (similar to the effect of real lens optics). In one study, blur was found to have no effect on judged distance [41], although another study testing nearer distances reported improved distance perception when blur was added [42]. Another study reported worse depth perception in the presence of blur, but paradoxically better performance on a task involving depth perception [43]. Of course, manipulating image blur is not identical to manipulating image resolution, so caution is warranted when interpreting these results in the context of the current project.

Studies manipulating graphics quality have compared high-quality surface textures (e.g., photographs taken from a real environment) with lower-quality textures containing less high-frequency detail and lacking in realism. Verbal judgments of distance were found to be shorter when viewing low-quality compared to high-quality textures [44], although no effect was found when using walking judgments [45]. These results are intriguing, but manipulating texture quality is not the same as manipulating resolution. Even low-quality textures presented through relatively low resolution can provide good texture cues to establish information about surface position and orientation.

HMD weight may also contribute to underperception of distance in VR. Reducing real world FOV while simultaneously weighting the head caused distance underperception compared to unrestricted and unencumbered viewing, although performance was still better than that in an HMD with the same weight and FOV [36]. Adding weight to a lighter HMD also caused underperception of distance compared to the unweighted HMD [22]. One proposed mechanism for the effect of HMD weight is that perceived distance 
depends partly on the perceived angular declination of the target [46], and a heavy HMD could bias perceived head angle during viewing [36].

The user or the researcher often adjusts eye relief and interpupillary distance in the process of fitting the HMD to the user's head. For example, a user might change the face pad to be thinner or thicker (e.g., to accommodate glasses), thus slightly changing the distance between their eyes and the HMD optics. Changing the eye relief in this way affects FOV and therefore pixel density. Interpupillary distance is also adjustable, which can impact the extent of binocular overlap of the images presented to the two eyes. Areas of binocular overlap essentially benefit from increased resolution, since the two eyes receive slightly different views of the same scene. However, these factors that are under the control of the user or the researcher are not typically reported in research publications. The current study therefore uses available HMD specifications without attempting to account for changes made for or by individual users.

In summary, there is some evidence that HMD FOV and weight affect perceived distance in VR, but limited evidence for the effect of resolution. These three factors are widely reported in the technical specifications of HMDs, and are therefore the primary predictor variables investigated in the meta-analysis. There are certainly other HMD characteristics beyond FOV, weight, and pixel density that might affect perceived distance. For example, different types of lenses in the HMD change the focal distance of the eye and affect other optical characteristics of the display, such as pincushion distortion [47]. Yet, there is limited experimental research on these variables, and HMD manufacturers do not systematically report these details. Therefore, the focus of the current project is on the potential influence of HMD FOV, weight, and resolution on perceived egocentric distance in VR.

\subsection{Overview of the meta-analysis}

Meta-analysis was undertaken with two primary goals. First, meta-analysis provides a summary of distance perception accuracy across all relevant studies as a whole, as well as separate summaries for frequently used HMDs. This is important because heterogeneity exists even among studies using the same HMD. In an extreme example, reports of mean distance judgments using the HTC Vive range from $66 \%$ [22] to $102 \%$ [27] of object distance. Second, metaanalysis indicates the relationship between perceived egocentric distance and technical characteristics of HMDs.

\section{Method}

\subsection{Literature search}

Relevant literature was identified by searching for keywords "distance perception," "distance judgment," "perceived distance" or "judged distance" in conjunction with "virtual reality" or "virtual environment" in the PsychInfo, PubMed, and Web of Science databases. The PsychInfo search allowed for up to two intervening words between the keywords, but this option was not available for the other searches. Literature was also added through hand-selection by conducting

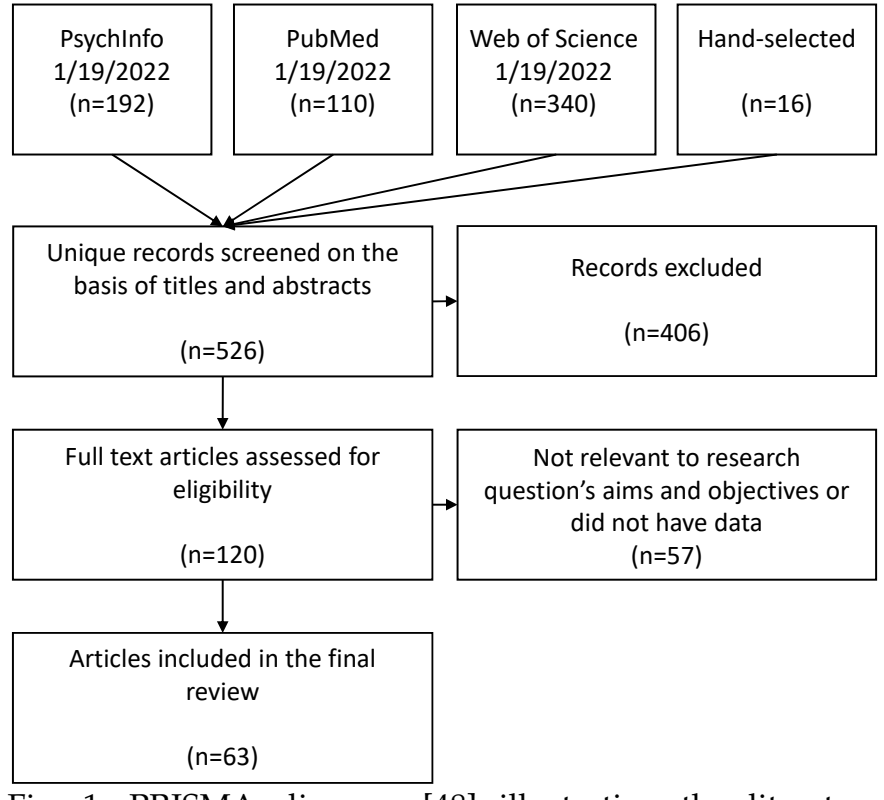

Fig. 1: PRISMA diagram [48] illustrating the literature search, screening, and selection process.

similar searches on Google Scholar, examining reference lists of relevant papers, and using domain knowledge. This resulted in 526 unique entries that were examined further using inclusion and exclusion criteria. Figure 1 shows a PRISMA [48] diagram summarizing the search process.

\subsection{Inclusion and exclusion criteria}

Studies were included/excluded using the following criteria:

Inclusion criteria:

- Measured perception of absolute egocentric distance in a virtual environment.

- Stimuli were presented through an HMD.

- Distances tested were primarily located in "action space" [13] (arm's reach to 30 meters).

Exclusion criteria:

- Distances tested were primarily located outside of action space [13] (e.g., within arm's reach).

- Did not measure perception of absolute egocentric distance (e.g., studies that measured relative egocentric distance using a bisection task or matching task, which can reflect whether space perception is linear but not whether it is under- or overperceived).

- Perception occurred through a non-visual sense (e.g., auditory distance perception).

- Used a display other than an HMD (e.g., smartphone, boom-mounted, CAVE, augmented reality).

- Data collected from a patient population (e.g., individuals with Parkinson's).

When possible, only the baseline condition was included from experiments that manipulated viewing conditions intended to modify perceived distance (e.g., manipulating eye height, increasing HMD weight, reducing HMD FOV, or removing stereo cues). However, the few studies lacking such baseline conditions were still included with the goal of including as many relevant studies as possible. 


\subsection{Coding}

Included studies were coded for means and standard deviations of judged distance as a percentage of actual distance. In cases where data were presented as a function of actual distance, the data were averaged across actual distance so that judged distance was summarized by a single mean and standard deviation. These data were sometimes reported in the text or in a table, but often had to be extracted from figures through digital measurement. Authors were contacted in cases where insufficient information was provided in the paper.

Studies were also coded for the HMD used, the number of participants, the type of distance judgment made (e.g., verbal, blind walking), and the minimum and maximum distances that were tested. Occasionally the same participants made multiple judgement types (e.g., verbal judgments and blind walking judgments) or experienced multiple conditions (e.g., two HMDs). These occurrences were coded so that the covariance between the repeated measures could be accounted for in the analysis. Technical characteristics of HMDs used in each study were identified through internet searches. Manufacturer specifications were used when provided, but in many cases these details had to be obtained through research reports and websites cataloging these details. The focus of this search was on HMD weight (in grams), FOV (in degrees), and resolution (in pixels/degree).

\subsection{Analysis}

A multi-model inference approach [50] was followed to address the research questions about the influence of HMD technical characteristics on perceived distance. Rather than searching for an individual regression model that best describes the data, multi-model inference considers all models given the various possible combinations of predictor variables. Models are then evaluated using a criterion, in this case Akaike information criterion (AIC), which rewards goodness of fit and model simplicity. The best model can then be chosen based on its AIC. Furthermore, the relative importance of each predictor is given by the sum of the relative evidence weights of all models in which the predictor appears. Importance can range from 0 to 1 , such that a predictor appearing in many models with large weights will receive a high importance value and a predictor that only appears in models with small weights will receive a low importance value. This multi-model inference approach is well suited to exploratory research lacking a priori predictions about specific models.

Although publication bias is often a risk in metaanalysis, it is not of significant concern in this study. Effect sizes describing comparison between two groups or conditions can be affected by publication bias (i.e., statistically significant comparisons are more likely to be published), but the current study analyzed judged distance directly. There is no logical reason why mean judged distance, which is essentially a descriptive statistic, would be affected by publication bias. Due to the long history of research documenting underperception in VR, reports of accurate distance perception seem likely to be as interesting as reports of underperception.
TABLE 1: HMDs included in the meta-analysis along with citations to the papers that contributed data.

\begin{tabular}{ll}
\hline HMD & Publications included \\
\hline Virtual Research V4 & {$[53]$} \\
Virtual Research FS5 & {$[54]$} \\
Virtual Research V8 & {$[46],[55],[56],[57]$} \\
nVision Datavisor HiRes & {$[45],[58],[59]$} \\
\hline nVisor SX60 & {$[22],[23],[44],[47],[60],[61],[62],[63]$,} \\
& {$[64],[65],[66],[67],[68],[69],[70],[71]$,} \\
& {$[36],[72],[73],[74]$} \\
eMagin 3DVisor Z800 & {$[75]$} \\
\hline Fakespace Wide 5 & {$[37]$} \\
nVisor ST & {$[76],[77],[78],[79]$} \\
nVisor ST50 & {$[80],[81],[82]$} \\
nVisor SX111 & {$[22],[83],[84],[85],[86]$} \\
\hline Sensics zSight & {$[41],[87]$} \\
Sensics xSight & {$[88]$} \\
Oculus Rift DK1 & {$[22],[28]$} \\
Oculus Rift DK2 & {$[6],[22],[23],[25],[30],[41],[89],[90]$} \\
\hline HTC Vive & {$[22],[24],[27],[31],[32],[33],[91]$} \\
HTC Vive Pro & {$[92]$} \\
Oculus Rift CV1 & {$[22],[26],[31],[33],[93]$} \\
Pimax 5k Plus & {$[29],[38]$} \\
\hline Oculus Quest & {$[94]$} \\
Oculus Quest 2 & {$[94]$}
\end{tabular}

Meta-analyses were conducted using the metafor package (version 3.0-2) with R Statistical Software (version 4.1.1). Data were weighted within the meta-analytic models based on the sample size and variance associated with each mean. Treating repeated measures (e.g., if the same participants judged distance in two HMDs) as if they were independent would artificially increase precision in the estimate [51]. Therefore, cases in which repeated measures were included were treated as a nested variable [52].

\section{Results}

The final sample included 137 data points involving 20 unique HMDs, and these data were extracted from 61 articles (see Table 1). Some articles contributed multiple data points when they presented multiple relevant conditions, experiments, or dependent measures. The data are available on the Open Science Framework [49].

Visual inspection along with a leave-one-out analysis was conducted to detect potential outliers. It was determined that none of the data had excessive influence on the pooled result, so all data were retained.

The forest plot in Figure 2 shows all of the 137 data points included in the analysis, sorted by the year in which the HMD was manufactured. An intercept-only regression model indicated that mean judged distance across all studies was $73.48 \%$ of actual distance, 95\% CI [71.17, 75.80]. Figure 3 shows judged distance summarized separately by HMD for those HMDs with three or more contributing studies.

The $\mathrm{I}^{2}$ index calculated using the entire data set was $93.8 \%$, indicating that considerable heterogeneity exists between studies. Multi-model inference was conducted to further explore whether the heterogeneity was related to characteristics of the HMD. Before proceeding with multi-model inference, potential predictor variables were centered and then inspected for multicollinearity by calculating the variance inflation factor (VIF) for each variable. HMD weight, horizontal FOV, vertical FOV, horizontal pixel density, and vertical pixel density were entered into a random effects linear meta-regression model for this purpose. 


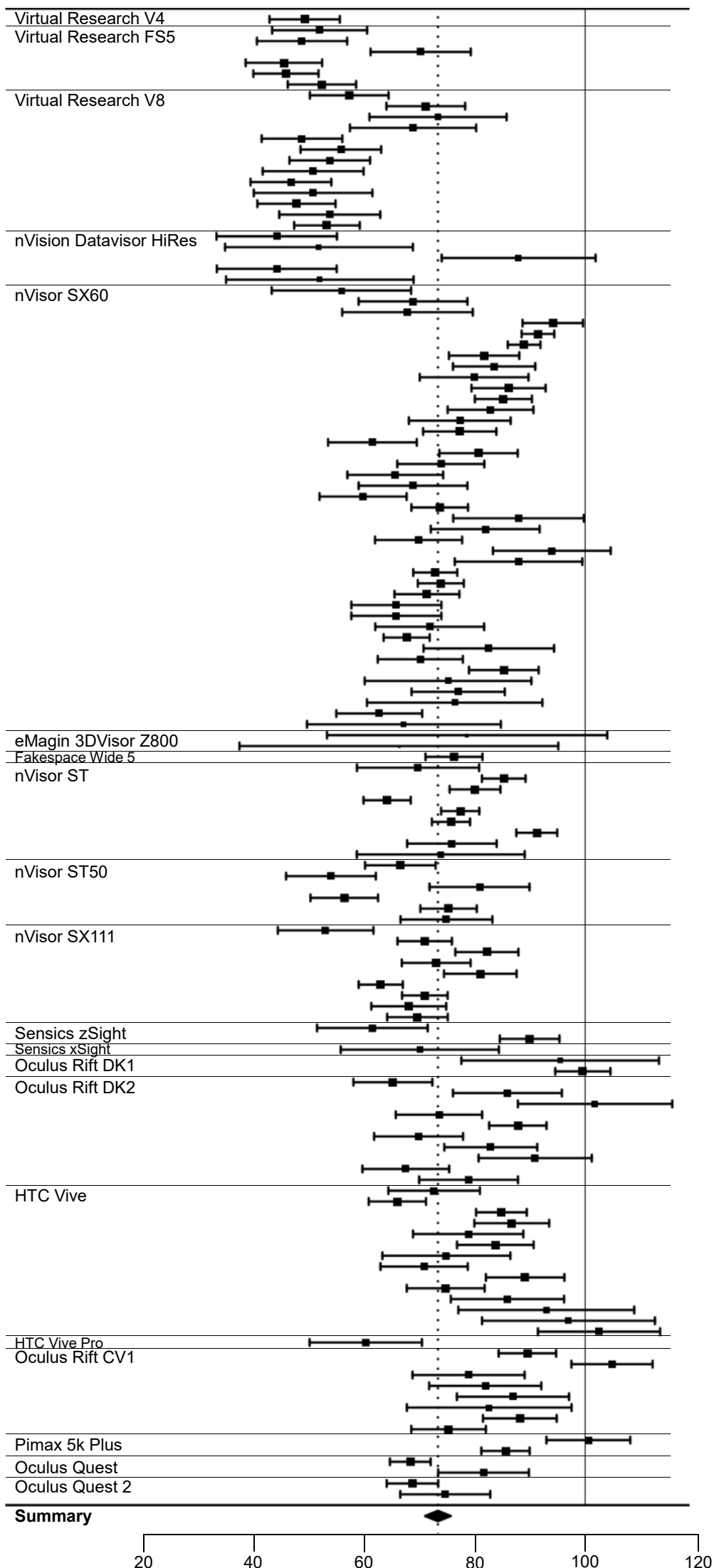

Fig. 2: Means and 95\% CI for all studies included in the meta-analysis. Labels indicate the HMD used in each study. Data are sorted by the year in which the HMD was first produced from oldest (top) to newest (bottom). Data provided on the Open Science Framework [49] can be used to connect data points to specific studies. The dotted vertical line represents the meta-analytic mean across all studies, and the solid vertical line at 100 represents perfect performance. 


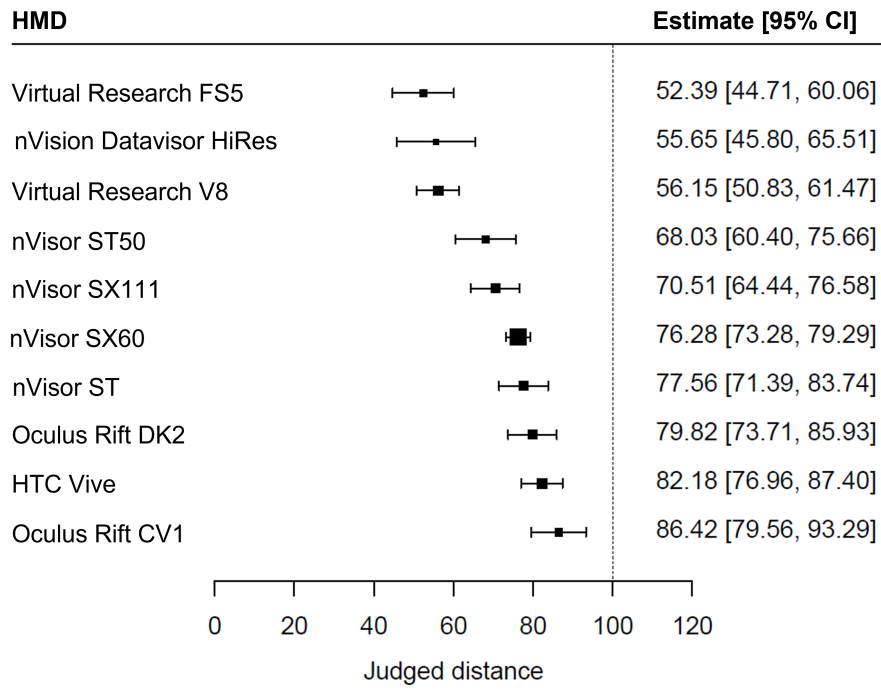

Fig. 3: Forest plot showing meta-analytic estimates of judged distance and 95\% CI for HMDs with at least three studies. Square size represents the precision of the estimate. Data are sorted by mean judgment. The vertical line at 100 represents perfect performance.

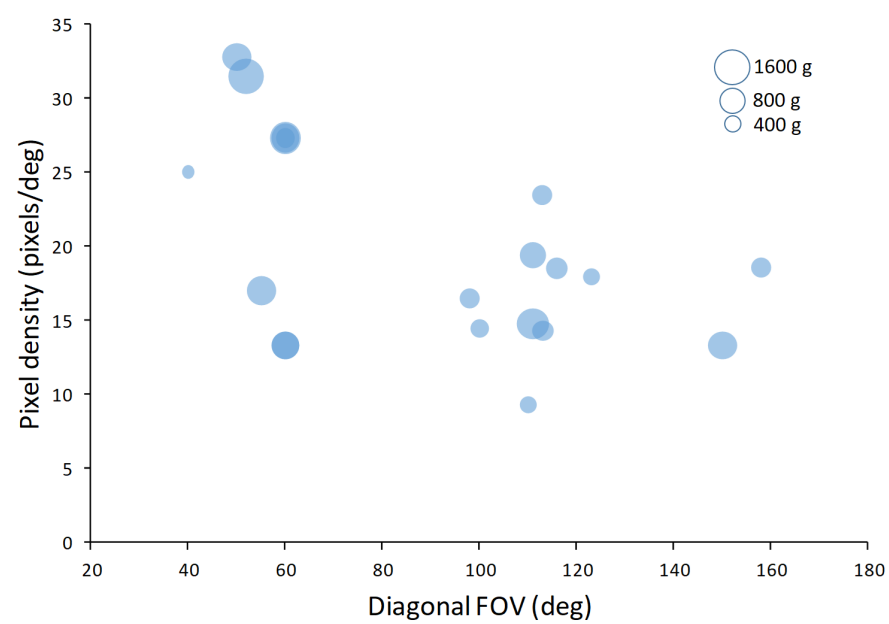

Fig. 4: Technical specifications of the HMDs included in the meta-analysis. Point size represents HMD weight. HMD names and other details can be found on the Open Science Framework [49].

Horizontal FOV, vertical FOV, horizontal pixel density, and vertical pixel density all produced unacceptably high VIF values $(>5.0)$ reflecting their relatedness to one or more other predictors. This is not surprising because horizontal and vertical display elements (FOV and resolution) are closely connected. Therefore, multiple variables were combined to create new variables representing diagonal FOV and diagonal pixel density. When possible, diagonal FOV was taken from manufacturer specifications or research reports, since FOV is not always rectangular. Entering these predictors along with weight into the meta-regression model produced acceptable VIF values (1.92, 1.86, and 1.46 for diagonal FOV, diagonal pixel density, and weight, respectively). These technical HMD characteristics are shown graphically in Figure 4, where each point represents a unique HMD.
Multi-model inference was conducted with diagonal FOV, diagonal pixel density, and weight entered as predictor variables and judged distance (as a percentage of actual distance) as the outcome variable. Models tested were all random effects regression models with repeated measures treated as a nested variable. The analysis included main effects and two-way interactions. The three-way interaction was not tested because the number of included studies was insufficient to meaningfully interpret such an interaction. The various combinations of predictor variables created 64 unique random effects regression models.

The model with the best (lowest) AIC value included three significant main effects corresponding to the three predictors (FOV, pixel density, and weight), as well as significant interactions between FOV and weight and between pixel density and weight. This model has an Akaike weight of .313 , meaning that there is a $31.3 \%$ chance that it is the best model of all models considered, and it explains $41.59 \%$ of the heterogeneity in the data. Model details are shown in Table 2. The next-best model has an Akaike weight of .145, which is considerably less than the best model. The list of all models below a cumulative Akaike weight of 95\% [95], [96] is shown in Table 3.

Model-averaged parameter estimates (i.e., weighted averages of model coefficients across all possible models) are shown in Table 4, sorted by parameter importance (i.e., the sum of the evidence weights across all models). All three main effects have high importance values, whereas the importance values for the three interactions are lower than those for the main effects and also below the recommended threshold of 0.8 [97]. This suggests that the two significant interactions in the best model (FOV by weight as well as pixel density by weight) are of limited importance. The third interaction (FOV by pixel density) is not considered further, since it is not a factor in the best model and is of lower importance across all models.

To summarize the results of the multi-model inference analysis, judged distance was negatively associated with weight (Figure 5), positively associated with diagonal FOV (Figure 6, top panel), and positively associated with pixel density (Figure 7, top panel). Two interactions were significant in the best model, but are of lower importance when considered across all possible models. The first interaction, between FOV and weight, is visualized in Figure 6. There is a sizeable gap in weight at around 700 grams (see Figure 6), so this is a sensible place to split the data to examine the interaction. Light HMDs (Figure 6, middle panel) showed no relationship between judged distance and FOV, $\beta=.093$, $95 \%$ CI $[-.123, .310]$, whereas heavy HMDs (Figure 6, bottom panel) produced a significant positive association between judged distance and FOV, $\beta=.324,95 \%$ CI [.179, .470]. The second interaction, between pixel density and weight, is shown in Figure 7. As with the previous interaction, the data were split into two groups based on HMD weight. Light HMDs (Figure 7, middle panel), showed no relationship between judged distance and pixel density, $\beta=-.673,95 \% \mathrm{CI}$ [-1.603, .257], whereas heavy HMDs (Figure 7, bottom panel) showed a significant positive association between judged distance and pixel density, $\beta=1.373,95 \%$ CI $[.952,1.793]$. 
TABLE 2: Details of the best-fitting regression model examining the effect of HMD characteristics on judged distance in VR. Variables assessed are diagonal field of view (FOV), diagonal pixel density, and HMD weight.

\begin{tabular}{lrrrrrr}
\hline Variable & Coefficients & Standard error & Z value & p value & Lower 95\% & Upper 95\% \\
\hline Intercept & 73.547 & 1.134 & 64.882 & $<.0001$ & 71.325 & 75.768 \\
FOV & 0.284 & 0.050 & 5.692 & $<.0001$ & 0.186 & 0.382 \\
Pixel density & 1.032 & 0.193 & 5.335 & $<.0001$ & 0.653 & 1.410 \\
\hline Weight & -0.017 & 0.004 & -4.392 & $<.0001$ & -0.025 & -0.010 \\
FOV ${ }^{*}$ Weight & 0.001 & 0.000 & 2.669 & 0.0076 & 0.000 & 0.001 \\
Pixel density * Weight & 0.002 & 0.001 & 3.021 & 0.0025 & 0.001 & 0.004 \\
\hline
\end{tabular}

Test of moderators: $\mathrm{QM}(\mathrm{df}=5)=80.693, \mathrm{p}<.0001$

Test for residual heterogeneity: $\mathrm{QE}(\mathrm{df}=131)=1312.697, \mathrm{p}<.0001$

$\mathrm{R}^{2}=41.59 \%$

$\mathrm{I}^{2}=89.49 \%$

$\operatorname{tau}^{2}=98.982(\mathrm{SE}=14.192)$
Log likelihood $=-522.605$

$\mathrm{AIC}=1059.209$

$\mathrm{AIC}_{\mathrm{c}}=1060.077$

$\mathrm{BIC}=1079.649$

TABLE 3: The best-ranked models (those with highest Akaike weight up to a cumulative weight of 0.95) examining the effect of HMD characteristics on judged distance in VR. Variables assessed are diagonal field of view (FOV), diagonal pixel density (Pixel) and HMD weight.

\begin{tabular}{|c|c|c|c|}
\hline Candidate model & $\mathrm{AIC}_{\mathrm{c}}$ & $\mathbf{w}_{\mathbf{i}}$ & acc $\mathbf{w}_{\mathbf{i}}$ \\
\hline FOV + Pixel + Weight + FOV*Weight + Pixel ${ }^{*}$ Weight & 1059.633 & 0.313 & 0.313 \\
\hline FOV + Pixel + Weight + FOV*Pixel & 1061.173 & 0.145 & 0.458 \\
\hline FOV + Pixel + Weight & 1061.508 & 0.123 & 0.581 \\
\hline FOV + Pixel + Weight + FOV ${ }^{*}$ Weight + Pixel ${ }^{*}$ Weight + FOV*Pixel & 1061.693 & 0.112 & 0.692 \\
\hline FOV + Pixel + Weight + Pixel ${ }^{*}$ Weight & 1062.417 & 0.078 & 0.770 \\
\hline FOV + Pixel + Weight + FOV*Pixel + Pixel $^{*}$ Weight & 1063.094 & 0.055 & 0.826 \\
\hline FOV + Pixel + Weight + FOV ${ }^{*}$ Weight + FOV*Pixel & 1063.325 & 0.049 & 0.875 \\
\hline FOV + Pixel + Weight + FOV $*$ Weight & 1063.488 & 0.046 & 0.921 \\
\hline Pixel + Weight + FOV*Pixel & 1064.572 & 0.026 & 0.947 \\
\hline
\end{tabular}

TABLE 4: Model-averaged parameter estimates (i.e., weighted averaged across all possible models), sorted by importance.

\begin{tabular}{lrrrrrr}
\hline Variable & Coefficients & Standard error & Z value & Lower 95\% & Upper 95\% & Importance \\
\hline Intercept & 72.814 & 1.949 & 37.354 & 68.993 & 76.634 & 1.000 \\
Weight & -0.016 & 0.004 & -4.006 & -0.024 & -0.008 & 0.999 \\
Pixel density & 0.949 & 0.310 & 3.060 & 0.341 & 1.557 & 0.969 \\
\hline FOV & 0.238 & 0.095 & 2.497 & 0.051 & 0.425 & 0.930 \\
Pixel density * Weight & 0.001 & 0.001 & 0.840 & -0.001 & 0.003 & 0.579 \\
FOV * Weight & 0.000 & 0.000 & 0.769 & 0.000 & 0.001 & 0.541 \\
FOV * Pixel density & -0.007 & 0.013 & -0.509 & -0.032 & 0.019 & 0.440
\end{tabular}

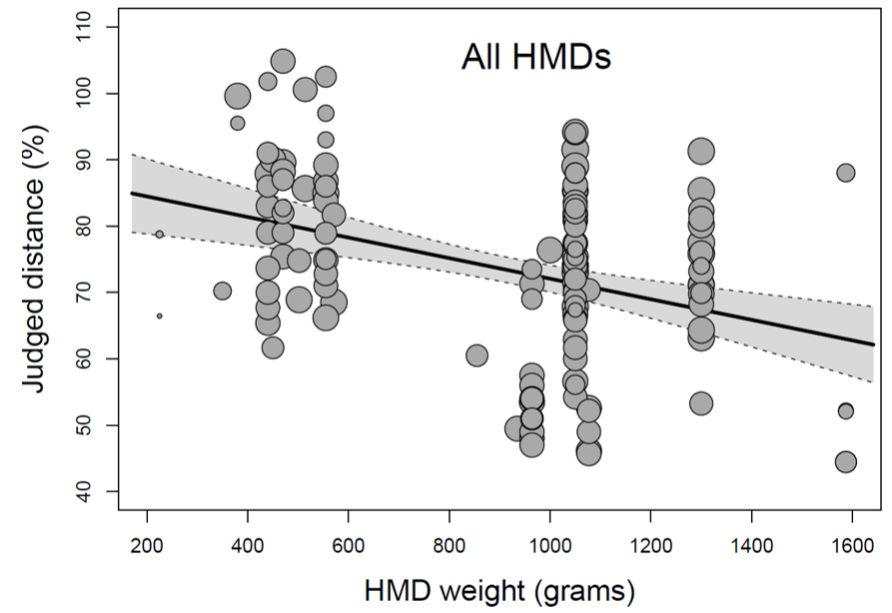

Fig. 5: Scatter plots showing the relationship between judged distance and HMD weight for all HMDs. Point size is proportional to weight in the meta-analysis.

\section{Discussion}

There were two primary goals of this meta-analysis. The first goal was to summarize research on perceived egocentric distance in VR. To that end, the analysis indicates that perceived distance across the entire literature is $73.48 \%$ of actual distance. However, the analysis also indicates that a single value is not the best way to represent this literature. The forest plot in Figure 2 is sorted (from top to bottom) by the year in which the HMD was first produced. Careful examination of these data reveal a trend toward greater accuracy among more modern HMDs (i.e., judged distance shifts rightward from top to bottom in the figure). Analysis of individual HMDs that were used in 3 or more studies (Figure 3) supports this conclusion. Three HMDs that were first produced in the 1990s through 2000, the Virtual Research FS5, Virtual Research V8, and nVision DataVisor HiRes, are associated with distance judgments of 52, 56, and $56 \%$, respectively. The nVisor SX60, first produced in 2003 and used in a large number of studies on this topic, is associated with distance judgments of $76 \%$, which is a considerable improvement over the prior generation. HMDs 

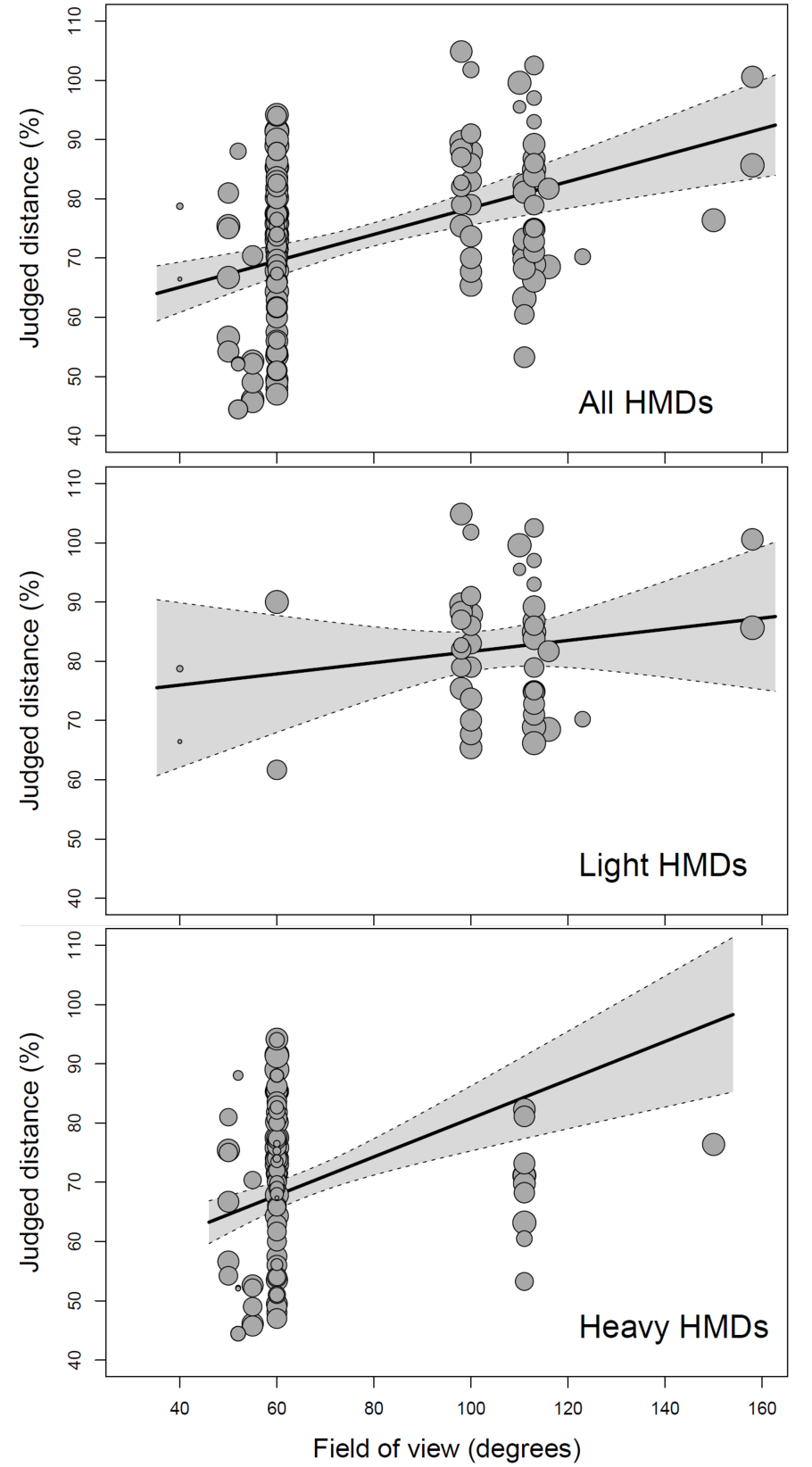

Fig. 6: Scatter plot showing the relationship between judged distance and HMD field of view for all HMDs (top), light HMDs (middle), and heavy HMDs (bottom). Point size is proportional to weight in the meta-analysis.

produced later in the 2000s, the nVisor ST, nVisor ST50, and nVisor SX111, are associated with distance judgments of 78,68 , and $71 \%$, respectively, indicating no notable improvement in perceived distance compared to the nVisor SX60. Consumer-oriented HMDs first sold in the 2010s, the Oculus Rift DK2, Oculus Rift CV1, and HTC Vive, are associated with distance judgments of 80,86 , and $82 \%$, respectively, indicating small but reliable improvement in judged distance compared to the prior generation of HMD.

Some HMDs produced intriguing results but were not presented in the preceding description because they were only used in one or two studies. The Oculus Rift DK1 produced impressively accurate distance judgments in two studies (96 and 100\% as reported by [22] and [28], respec-
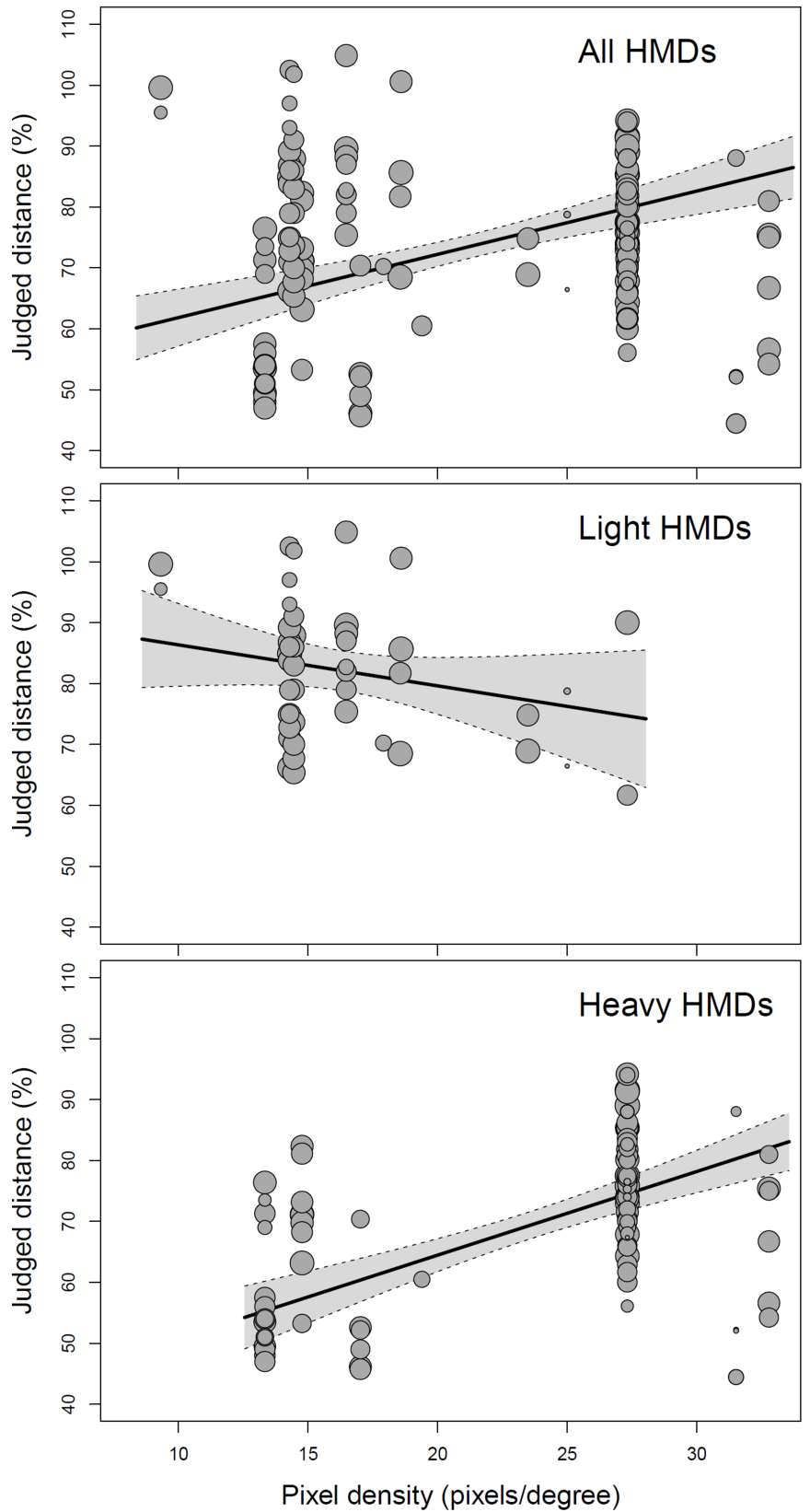

Fig. 7: Scatter plots showing the relationship between judged distance and HMD pixel density for all HMDs (top), light HMDs (middle), and heavy HMDs (bottom). Point size is proportional to weight in the meta-analysis.

tively). Also impressive, the Pimax 5k Plus, which boasts a very wide FOV (almost 160 degree diagonal), produced distance judgments of $86 \%$ and $100 \%$ in two studies [29], [38]. Yet, caution is warranted when interpreting these results based on limited evidence. The reported means may be near the true mean, but reports also exist of highly accurate distance judgments in the Oculus Rift DK2 [25], Oculus Rift CV1 [26], and HTC Vive [27], yet meta-analysis of all studies using those headsets reveals a considerably lower mean. Therefore, these intriguing results based on limited evidence should be followed up with additional research to confirm whether judged distance is superior in these HMDs.

The second goal of the meta-analysis was to evaluate potential relationships between perceived distance and tech- 
nical characteristics of HMDs, specifically FOV, weight, and resolution. The included studies covered 20 different HMDs, varying widely in their technical specifications. This analysis revealed significant associations with and interactions between FOV, weight, and pixel density. All three main effects were significant. More accurate distance judgments were associated with wider field of view, lower weight, and greater pixel density. However, each main effect was contextualized by at least one interaction, discussed in further detail below.

The interaction between FOV and weight (significant in the best model, although of limited importance when considered across all models) indicated that FOV only significantly predicted judged distance among heavier HMDs, and that no relationship existed between judged distance and FOV among lighter HMDs. The underlying cause of this interaction is unclear, but some speculation is possible. Whereas heavier HMDs varied somewhat in FOV, most of the lighter HMDs were clustered in FOV around 100 degrees, with relatively sparse sampling at lower and higher FOV. Therefore, it is possible that the lack of association between judged distance and FOV among lighter HMDs is due to insufficient FOV variation in the data set. The few studies that have used lighter HMDs at the lower (e.g., the eMagin 3DVisor Z800) and upper (e.g., Pimax 5k Plus) ends of the FOV spectrum appear to indicate a subtle positive impact of FOV. New HMDs are likely to be light weight and have higher FOV, and it is therefore possible that additional sampling in this space would reveal a significant relationship between judged distance and FOV. It is also notable that modern HMDs are generally lighter and have higher FOV compared to older HMDs. It is therefore possible that third variables not included in the regression analysis would explain these findings.

The interaction between pixel density and HMD weight (significant in the best model, but of limited importance across all models) indicated that pixel density only significantly predicted judged distance among heavier HMDs, and that no relationship existed between judged distance and pixel density among lighter HMDs. There is a good distribution of pixel density in the data set among both light and heavy HMDs, so range restriction is not a likely explanation for the lack of association among lighter HMDs. At the extreme ends of pixel density among lighter HMDs, the Oculus DK1 possesses very low pixel density but produced relatively accurate distance judgments, whereas the Sensics zSight possesses high pixel density but produced moderate underperception of distance. Although pixel density is not an important predictor of judged distance among lighter HMDs, future HMDs are still likely to emphasize higher resolution displays due to consumer demand for better image quality. It will be interesting to see how new higher resolution HMDs perform in comparison to the lighter HMDs included in this analysis.

How do the findings from the meta-regression fit with experimental evidence? Multiple studies have shown that experimentally reducing HMD FOV causes further underperception of distance [22], [29], [37], [38]. These results are generally consistent with the findings of the meta-analysis, which found a significant positive association between FOV and judged distance. The only study to experimentally add weight to an HMD found that it caused further underperception of distance [22]. This finding is consistent with the findings of the meta-analysis, particularly since the HMD used in the aforementioned experiment had a relatively wide FOV. Only a few studies have explored the effect of image resolution on judged distance, and the results have produced mixed evidence [34], [39], [40]. In contrast, the meta-analysis points to a significant role of resolution (operationalized as pixel density) in perceived distance, especially among heavier HMDs. Thus, the experimental findings on HMD FOV and weight are generally consistent with the conclusions of the meta-analysis, whereas the experimental findings on HMD resolution are limited but seem to contradict the findings of the meta-analysis. Therefore, one conclusion from this meta-analysis is the need for more experimental research exploring the effect of HMD resolution on perceived distance.

One challenge in meta-analyzing the literature on distance perception in VR is that HMD characteristics do not vary randomly. Newer HMDs often make simultaneous improvements in FOV, resolution, and weight over the prior generation. Along with those improvements come other improvements that are infrequently reported and more difficult to measure, such as optical quality, display brightness/contrast, and ergonomics. Correlations among HMD weight, FOV, resolution, and other HMD characteristics are therefore expected. Although multicollinearity was not a concern among the variables included in the meta-analysis, this does not mean that the analysis is free from bias caused by other variables that change over time. Correlations between the release year of the HMD and the variables considered in the model were $-.28,-.63$, and .78 for pixel density, weight, and FOV, respectively. These values indicate that conclusions about HMD weight and FOV are most vulnerable to third variables (e.g., optics quality) that also change over time as HMD technology advances. Details of the research studies might also fluctuate over time. For example, studies testing two earlier HMDs (the Virtual Research FS5 [54] and nVision DataVisor HiRes [45], [58], [59]) tested relatively long distances (maximum distance around 13 meters) compared to other studies (average maximum distance across all studies $=7.3$ meters). Likewise, improvements to the virtual environments occur over time. For example, newer VEs typically contain realistic textures, shadows, and reflections that were missing from early studies. New HMDs are also much more portable due to onboard processors that no longer require a dedicated graphics computer, and due to onboard tracking solutions that no longer require carefully-calibrated cameras placed around the room. These improvements allow researchers to move their experiments outside the lab into larger physical spaces, which appears to impact distance judgments [27]. In sum, newer HMDs differ from older HMDs in many ways that are difficult to characterize and account for in a metaanalysis. It is therefore possible that the effects of HMD FOV, resolution, and weight identified in the meta-analysis are partially attributable to other advances that are not captured in the meta-analysis.

Another limitation of the meta-analysis is that the 20 HMDs included do not provide even sampling along the technical dimensions of FOV, weight, and resolution. For 
example, HMD FOV clusters in two bands, one around 5060 degrees and one around 120-150 degrees, with very few HMDs outside those bands. Lack of diversity in technical specifications is a clear limitation of the meta-regression, although continued research with more HMDs will increase diversity.

Future meta-analysis of research on perceived distance in personal space (within reaching distance [13]) could prove fruitful. The current meta-analysis excluded such studies because it is not clear that underperception is a persistent problem within personal space. Yet, careful consideration of experimental details (e.g., VE characteristics, HMD characteristics, and response methods) may reveal systematic differences in perception of absolute distance within personal space.

Although HMD technical factors explain a significant amount of the variance in judged distance, substantial unexplained variance remains. For example, of the 41 studies conducted using the nVisor SX60 (the most studies associated with any of the HMDs), mean distance judgments range from $56 \%$ to $94 \%$. The differences among these studies cannot be attributable to HMD specifications, since all used the same HMD. Some of the variation could be caused by small sample sizes and correspondingly poor estimates of the mean (the modal sample size among those studies is 10 participants), but other factors likely contribute to the variance across studies. Some of these factors have already been explored experimentally, such as the impact of a selfavatar [30], [66], [71], [73] or a VE that matches a previously experienced real environment [61], [75], [83]. Other factors might also be important to consider, such as the quality of the VE (e.g., realism, or presence of shadows), training protocols (e.g., whether participants were calibrated to making verbal judgments in the real world prior to entering the virtual world), and the method of distance judgment. These differences may help to further explain variance across studies, even those using the same HMD. In order to reduce variance across similar studies (e.g., multiple studies that use the same HMD), it could be beneficial for the field to develop a set of best practices for research on distance perception in VR.

Distance perception is more accurate in modern HMDs compared to prior generations. As judged distance approaches perfect performance, it is important to consider the standard by which performance is evaluated. Many studies compare perceived distance in VR to that in a real environment, often using a carefully matched VE (e.g., [24], [25], [45]). However, many studies do not include such a real world comparison condition. Real world distance perception is often near $100 \%$ of actual distance, but this is not always the case (e.g., [24], [33], [91], [98]) and may depend on details of the environment and the research protocols. Researchers studying perceived distance in VR would therefore be wise to include real world controls when possible.

In summary, this project analyzed 137 reports of egocentric distance judgments in HMD-based VR. Meta-analysis was conducted to describe distance underperception across several HMDs. Although there is a trend toward improvement among modern HMDs, underperception persists even in HMDs currently being sold. Meta-regression revealed associations between judged distance and HMD properties. Wider FOV, lighter weight, and higher resolution were all associated with more accurate distance judgments. The impact of FOV and pixel density were more pronounced among heavier HMDs. It is possible that future improvements in HMD FOV, resolution, and weight will lead to further improvement in perceived distance. Future research on the topic is constrained by technical characteristics of available HMDs, but the recent availability of relatively light HMDs with very wide FOV create interesting opportunities for experimental research.

\section{REFERENCES}

[1] B. Bodenheimer, J. Meng, H. Wu, G. Narasimham, B. Rump, T. P. McNamara, T. H. Carr, and J. J. Rieser, "Distance estimation in virtual and real environments using bisection," in Proceedings of the 4th Symposium on Applied Perception in Graphics and Visualization, ser. APGV '07. New York, NY, USA: Association for Computing Machinery, 2007, p. 35-40.

[2] E. Peillard, T. Thebaud, J.-M. Normand, F. Argelaguet, G. Moreau, and A. Lécuyer, "Virtual objects look farther on the sides: The anisotropy of distance perception in virtual reality," in 2019 IEEE Conference on Virtual Reality and 3D User Interfaces (VR), 2019, pp. 227-236.

[3] Z. Li, J. Phillips, and F. H. Durgin, "The underestimation of egocentric distance: Evidence from frontal matching tasks," Attention, Perception, E Psychophysics, vol. 73, no. 7, pp. 2205-2217, 2011.

[4] H. Gagnon, S. Creem-Regehr, and J. Stefanucci, "Virtual room re-creation: A new measure of room size perception," in ACM Symposium on Applied Perception 2021, 2021, pp. 1-10.

[5] C. von Castell, D. Oberfeld, and H. Hecht, "Visual and postural eye-height information is flexibly coupled in the perception of virtual environments," Journal of Experimental Psychology: Human Perception and Performance, vol. 47, no. 8, pp. 1132-1148, 2021.

[6] M. Loyola, "The influence of the availability of visual cues on the accurate perception of spatial dimensions in architectural virtual environments," Virtual Reality, vol. 22, no. 3, pp. 235-243, 2018.

[7] N. C. Nilsson, S. Serafin, and R. Nordahl, "The effect of head mounted display weight and locomotion method on the perceived naturalness of virtual walking speeds," in 2015 IEEE Virtual Reality (VR), 2015, pp. 249-250.

[8] A. Bhargava, K. M. Lucaites, L. S. Hartman, H. Solini, J. W. Bertrand, A. C. Robb, C. C. Pagano, and S. V. Babu, "Revisiting affordance perception in contemporary virtual reality," Virtual Reality, vol. 24, no. 4, pp. 713-724, 2020.

[9] M. Geuss, J. Stefanucci, S. Creem-Regehr, and W. B. Thompson, "Can i pass? using affordances to measure perceived size in virtual environments," in Proceedings of the 7th Symposium on Applied Perception in Graphics and Visualization, ser. APGV'10. New York NY, USA: Association for Computing Machinery, 2010, p. 61-64.

[10] P. B. Kline and B. G. Witmer, "Distance perception in virtua environments: Effects of field of view and surface texture at near distances," Proceedings of the Human Factors and Ergonomics Society - 40th Annual Meeting, pp. 1112-1116, 1996.

[11] B. Witmer and P. Kline, "Judging perceived and traversed distance in virtual environments," Presence-Teleoperators and Virtual Environments, vol. 7, no. 2, pp. 144-167, 1998.

[12] B. G. Witmer and W. J. Sadowski, "Nonvisually guided locomotion to a previously viewed target in real and virtual environments," Human Factors, vol. 40, no. 3, pp. 478-488, 1998.

[13] J. E. Cutting and P. M. Vishton, "Perceiving layout and knowing distances: The integration, relative potency, and contextual use of different information about depth," in Perception of space and motion. Elsevier, 1995, pp. 69-117.

[14] C. Armbrüster, M. Wolter, T. Kuhlen, W. Spijkers, and B. Fimm, "Depth perception in virtual reality: distance estimations in periand extrapersonal space," Cyberpsychology $\mathcal{E}$ Behavior, vol. 11, no. 1, pp. 9-15, 2008.

[15] G. P. Bingham, "Calibration of distance and size does not calibrate shape information: Comparison of dynamic monocular and static and dynamic binocular vision," Ecological Psychology, vol. 17, no. 2, pp. 55-74, 2005. 
[16] S. A. Linkenauger, H. H. Bulthoff, and B. J. Mohler, "Virtual arm's reach influences perceived distances but only after experience reaching," Neuropsychologia, vol. 70, pp. 393-401, 2015.

[17] D. Mine, S. Kimoto, and K. Yokosawa, "Obstacles affect perceptions of egocentric distances in virtual environments," Frontiers in Virtual Reality, p. 153, 2021.

[18] A. Murgia and P. M. Sharkey, "Estimation of distances in virtual environments using size constancy," International Journal of Virtual Reality, vol. 8, no. 1, pp. 67-74, 2009.

[19] A. Naceri, R. Chellali, and T. Hoinville, "Depth perception within peripersonal space using head-mounted display," Presence: Teleoperators and Virtual Environments, vol. 20, no. 3, pp. 254-272, 2011.

[20] P. E. Napieralski, B. M. Altenhoff, J. W. Bertrand, L. O. Long, S. V. Babu, C. C. Pagano, J. Kern, and T. A. Davis, "Near-field distance perception in real and virtual environments using both verbal and action responses," ACM Trans. Appl. Percept., vol. 8, no. 3, aug 2011.

[21] J. P. Rolland, W. Gibson, and D. Ariely, "Towards quantifying depth and size perception in virtual environments," Presence: Teleoperators \& Virtual Environments, vol. 4, no. 1, pp. 24-49, 1995.

[22] L. E. Buck, M. K. Young, and B. Bodenheimer, "A comparison of distance estimation in HMD-based virtual environments with different HMD-based conditions," ACM Trans. Appl. Percept., vol. 15, no. 3, 2018.

[23] S. H. Creem-Regehr, J. K. Stefanucci, W. B. Thompson, N. Nash, and M. McCardell, "Egocentric distance perception in the oculus rift (dk2)," Proceedings of the ACM SIGGRAPH Symposium on Applied Perception, p. 47-50, 2015.

[24] J. W. Kelly, L. A. Cherep, and Z. D. Siegel, "Perceived space in the htc vive," ACM Trans. Appl. Percept., vol. 15, no. 1, 2017.

[25] I. T. Feldstein, F. M. Kolsch, and R. Konrad, "Egocentric distance perception: A comparative study investigating differences between real and virtual environments." Perception, vol. 49, no. 9, pp. $940-967,2020$.

[26] S. Ahn, S. Kim, and S. Lee, "Effects of visual cues on distance perception in virtual environments based on object identification and visually guided action," International Journal of Human-Computer Interaction, vol. 37, no. 1, pp. 36-46, 2021.

[27] J. Zhang, X. Yang, Z. Jin, and L. Li, "Distance estimation in virtual reality is affected by both the virtual and the real-world environments." i-Perception, vol. 12, no. 3, pp. 1-6, 2021.

[28] B. Li, R. Zhang, and S. Kuhl, "Minication affects action-based distance judgments in oculus rift HMDs," Proceedings of the ACM Symposium on Applied Perception, p. 91-94, 2014.

[29] S. Masnadi, K. P. Pfeil, J.-V. T. Sera-Josef, and J. J. LaViola, "Field of view effect on distance perception in virtual reality," 2021 IEEE Conference on Virtual Reality and 3D User Interfaces, Abstracts and Workshops, pp. 542-543, 2021.

[30] D. Valkov, J. Martens, and K. Hinrichs, "Evaluation of the effect of a virtual avatar's representation on distance perception in immersive virtual environments," 2016 IEEE Virtual Reality Conference, pp. 305-306, 2016.

[31] S. Aseeri, K. Paraiso, and V. Interrante, "Investigating the influence of virtual human entourage elements on distance judgments in virtual architectural interiors." Frontiers in robotics and AI, vol. 6, 2019.

[32] P. Maruhn, S. Schneider, and K. Bengler, "Measuring egocentric distance perception in virtual reality: Influence of methodologies, locomotion and translation gains," Plos One, vol. 14, no. 10, 2019.

[33] A. Peer, K. Ponto, M. Marchal, R. Teather, and B. Thomas, "Evaluating perceived distance measures in room-scale spaces using consumer-grade head mounted displays," 2017 IEEE Symposium on 3D User Interfaces, pp. 83-86, 2017.

[34] K.-M. Jaeae-Aro and L. Kjelldahl, "Effects of image resolution on depth perception in stereo and nonstereo images," in Stereoscopic Displays and Virtual Reality Systems IV, S. S. Fisher, J. O. Merritt, and M. T. Bolas, Eds., vol. 3012, International Society for Optics and Photonics. SPIE, 1997, pp. $319-326$.

[35] J. M. Knapp and J. M. Loomis, "Limited Field of View of HeadMounted Displays Is Not the Cause of Distance Underestimation in Virtual Environments," Presence: Teleoperators and Virtual Environments, vol. 13 , no. 5, pp. 572-577, 2004

[36] P. Willemsen, M. B. Colton, S. H. Creem-Regehr, and W. B. Thompson, "The effects of head-mounted display mechanical properties and field of view on distance judgments in virtual environments," ACM Trans. Appl. Percept., vol. 6, no. 2, 2009.

[37] J. A. Jones, J. E. Swan, and M. Bolas, "Peripheral stimulation and its effect on perceived spatial scale in virtual environments," IEEE
Transactions on Visualization and Computer Graphics, vol. 19, no. 4, pp. 701-710, 2013.

[38] S. Masnadi, K. Pfeil, J.-V. T. Sera-Josef, and J. LaViola, "Effects of field of view on egocentric distance perception in virtual reality," in CHI Conference on Human Factors in Computing Systems, ser. CHI '22. New York, NY, USA: Association for Computing Machinery, 2022.

[39] J. Ryu, N. Hashimoto, and M. Sato, "Influence of resolution degradation on distance estimation in virtual space displaying static and dynamic image," in 2005 International Conference on Cyberworlds (CW'05), 2005, pp. 8 pp. -50.

[40] A. Dey, G. Jarvis, C. Sandor, and G. Reitmayr, "Tablet versus phone: Depth perception in handheld augmented reality," in 2012 IEEE International Symposium on Mixed and Augmented Reality (ISMAR), 2012, pp. 187-196.

[41] E. Langbehn, T. Raupp, G. Bruder, F. Steinicke, B. Bolte, and M. Lappe, "Visual blur in immersive virtual environments: Does depth of field or motion blur affect distance and speed estimation?" in Proceedings of the 22nd ACM Conference on Virtual Reality Software and Technology. New York, NY, USA: Association for Computing Machinery, 2016, p. 241-250.

[42] M. Moehring, A. Gloystein, and R. Doerner, "Issues with virtual space perception within reaching distance: Mitigating adverse effects on applications using hmds in the automotive industry," in 2009 IEEE Virtual Reality Conference, 2009, pp. 223-226.

[43] M. A. Cidota, R. M. Clifford, S. G. Lukosch, and M. Billinghurst "Using visual effects to facilitate depth perception for spatial tasks in virtual and augmented reality," in 2016 IEEE International Symposium on Mixed and Augmented Reality (ISMAR-Adjunct), 2016, pp. $172-177$.

[44] B. R. Kunz, L. Wouters, D. Smith, W. B. Thompson, and S. H. Creem-Regehr, "Revisiting the effect of quality of graphics on distance judgments in virtual environments: A comparison of verbal reports and blind walking," Attention, Perception, \& Psychophysics, vol. 71, no. 6, pp. 1284-1293, 2009.

[45] W. B. Thompson, P. Willemsen, A. A. Gooch, S. H. Creem-Regehr, J. M. Loomis, and A. C. Beall, "Does the quality of the computer graphics matter when judging distances in visually immersive environments?" Presence, vol. 13, no. 5, pp. 560-571, 2004.

[46] R. Messing and F. H. Durgin, "Distance perception and the visual horizon in head-mounted displays," ACM Transactions on Applied Perception, vol. 2, no. 3, pp. 234-250, 2005.

[47] S. A. Kuhl, W. B. Thompson, and S. H. Creem-Regehr, "HMD calibration and its effects on distance judgments," ACM Trans. Appl. Percept., vol. 6, no. 3, 2009.

[48] D. Moher, A. Liberati, J. Tetzlaff, and D. G. Altman, "Preferred reporting items for systematic reviews and meta-analyses: the prisma statement," Annals of internal medicine, vol. 151, no. 4, pp. 264-269, 2009.

[49] J. W. Kelly, "Meta-analysis of distance perception in virtual reality," Feb 2022. [Online]. Available: https://osf.io/bpj8a/

[50] D. R. Anderson, Model based inference in the life sciences: A primer on evidence. New York: Springer, 2007.

[51] R. D. Riley, "Multivariate meta-analysis: the effect of ignoring within-study correlation," Journal of the Royal Statistical Society: Series A (Statistics in Society), vol. 172, no. 4, pp. 789-811, 2009.

[52] M. W.-L. Cheung, "A guide to conducting a meta-analysis with non-independent effect sizes," Neuropsychology review, vol. 29, no. 4, p. 387-396, 2019.

[53] J. A. Ehrlich, "The effect of viewing conditions on visual stress, sickness, and distance estimation in a helmet-mounted display," Dissertation Abstracts International: Section B: The Sciences and Engineering, 1999.

[54] J. M. Knapp, "The visual perception of egocentric distance in virtual environments," Dissertation Abstracts International: Section B: The Sciences and Engineering, 2001

[55] J. M. Foley, "Visually directed action," Journal of Vision, vol. 21, no. $5,2021$.

[56] A. R. Richardson and D. Waller, "Interaction with an immersive virtual environment corrects users' distance estimates," Human Factors, vol. 49, no. 3, pp. 507-517, 2007.

[57] D. Waller and A. R. Richardson, "Correcting distance estimates by interacting with immersive virtual environments: Effects of task and available sensory information," Journal of Experimental Psychology: Applied, vol. 14, no. 1, pp. 61-72, 2008. 
[58] P. Willemsen and A. Gooch, "Perceived egocentric distances in real, image-based, and traditional virtual environments," IEEE Virtual Reality 2002, Proceedings, pp. 275-276, 2002.

[59] P. Willemsen, A. A. Gooch, W. B. Thompson, and S. H. CreemRegehr, "Effects of stereo viewing conditions on distance perception in virtual environments," Presence-Teleoperators and Virtual Environments, vol. 17, no. 1, pp. 91-101, 2008.

[60] M. N. Geuss, J. K. Stefanucci, S. H. Creem-Regehr, and W. B. Thompson, "Effect of viewing plane on perceived distances in real and virtual environments," Journal of Experimental Psychology: Human Perception and Performance, vol. 38, no. 5, pp. 1242-1253, 2012.

[61] V. Interrante, B. Ries, and L. Anderson, "Distance perception in immersive virtual environments, revisited," in IEEE Virtual Reality Conference (VR 2006), 2006, pp. 3-10.

[62] V. Interrante, B. Ries, J. Lindquist, M. Kaeding, and L. Anderson, "Elucidating Factors that Can Facilitate Veridical Spatial Perception in Immersive Virtual Environments," Presence: Teleoperators and Virtual Environments, vol. 17, no. 2, pp. 176-198, 042008.

[63] S. A. Kuhl, W. B. Thompson, and S. H. Creem-Regehr, "Minification influences spatial judgments in virtual environments," in Proceedings of the 3rd Symposium on Applied Perception in Graphics and Visualization. New York, NY, USA: Association for Computing Machinery, 2006, p. 15-19.

[64] B. R. Kunz, S. H. Creem-Regehr, and W. B. Thompson, "Testing the mechanisms underlying improved distance judgments in virtual environments," Perception, vol. 44, no. 4, pp. 446-453, 2015.

[65] M. Leyrer, S. A. Linkenauger, H. H. Bülthoff, and B. J. Mohler, "Eye height manipulations: A possible solution to reduce underestimation of egocentric distances in head-mounted displays," ACM Transactions on Applied Perception, vol. 12, no. 1, pp. 1-23, 2015.

[66] M. Leyrer, S. A. Linkenauger, H. H. Bülthoff, U. Kloos, and B. Mohler, "The influence of eye height and avatars on egocentric distance estimates in immersive virtual environments," in Proceedings of the ACM SIGGRAPH Symposium on Applied Perception in Graphics and Visualization. New York, NY, USA: Association for Computing Machinery, 2011, p. 67-74.

[67] M. Leyrer, S. A. Linkenauger, H. H. Bulthoff, and B. J. Mohler, "The importance of postural cues for determining eye height in immersive virtual reality." PloS one, vol. 10, no. 5, 2015.

[68] B. J. Mohler, S. H. Creem-Regehr, and W. B. Thompson, "The influence of feedback on egocentric distance judgments in real and virtual environments," in Proceedings of the 3rd Symposium on Applied Perception in Graphics and Visualization. New York, NY, USA: Association for Computing Machinery, 2006, p. 9-14.

[69] T. D. Nguyen, C. J. Ziemer, T. Grechkin, B. Chihak, J. M. Plumert, J. F. Cremer, and J. K. Kearney, "Effects of scale change in distance perception in virtual environments," ACM Transactions on Applied Perception, vol. 8, no. 4, pp. 1-18, 2011.

[70] L. Phillips, B. Ries, V. Interrante, M. Kaeding, and L. Anderson, "Distance perception in npr immersive virtual environments, revisited," in Proceedings of the 6th Symposium on Applied Perception in Graphics and Visualization, ser. APGV '09. New York, NY, USA: Association for Computing Machinery, 2009, p. 11-14.

[71] L. Phillips, B. Ries, M. Kaeding, and V. Interrante, "Avatar self-embodiment enhances distance perception accuracy in nonphotorealistic immersive virtual environments," in 2010 IEEE Virtual Reality Conference (VR), 2010, pp. 115-1148.

[72] L. Phillips and V. Interrante, "A little unreality in a realistic replica environment degrades distance estimation accuracy," in 2011 IEEE Virtual Reality Conference, 2011, pp. 235-236.

[73] B. Ries, V. Interrante, M. Kaeding, and L. Anderson, "The effect of self-embodiment on distance perception in immersive virtual environments," in Proceedings of the 2008 ACM Symposium on Virtual Reality Software and Technology. New York, NY, USA: Association for Computing Machinery, 2008, p. 167-170.

[74] C. S. Sahm, S. H. Creem-Regehr, W. B. Thompson, and P. Willemsen, "Throwing versus walking as indicators of distance perception in similar real and virtual environments," ACM Transactions on Applied Perception, vol. 2, no. 1, pp. 35-45, 2005.

[75] F. Steinicke, G. Bruder, K. Hinrichs, and A. Steed, "Gradual transitions and their effects on presence and distance estimation," Computers $\mathcal{E}$ Graphics, vol. 34, no. 1, pp. 26-33, 2010.

[76] T. Y. Grechkin, T. D. Nguyen, J. M. Plumert, J. F. Cremer, and J. K. Kearney, "How does presentation method and measurement protocol affect distance estimation in real and virtual environments?" ACM Transactions on Applied Perception, vol. 7, no. 4, 2010.
[77] J. A. Jones, J. E. Swan, G. Singh, E. Kolstad, and S. R. Ellis, "The effects of virtual reality, augmented reality, and motion parallax on egocentric depth perception," in Proceedings of the 5th Symposium on Applied Perception in Graphics and Visualization, ser. APGV '08. New York, NY, USA: Association for Computing Machinery, 2008, p. 9-14.

[78] J. A. Jones, J. E. Swan, G. Singh, and S. R. Ellis, "Peripheral visual information and its effect on distance judgments in virtual and augmented environments," in Proceedings of the ACM SIGGRAPH Symposium on Applied Perception in Graphics and Visualization, ser. APGV '11. New York, NY, USA: Association for Computing Machinery, 2011, p. 29-36.

[79] R. Zhang, A. Nordman, J. Walker, and S. A. Kuhl, "Minification affects verbal- and action-based distance judgments differently in head-mounted displays," ACM Trans. Appl. Percept., vol. 9, no. 3, 2012.

[80] J. W. Kelly, W. W. Hammel, Z. D. Siegel, and L. A. Sjolund, "Recalibration of perceived distance in virtual environments occurs rapidly and transfers asymmetrically across scale," IEEE Transactions on Visualization and Computer Graphics, vol. 20, no. 4, pp. 588-595, 2014.

[81] J. W. Kelly, W. Hammel, L. A. Sjolund, and Z. D. Siegel, "Frontal extents in virtual environments are not immune to underperception," Attention, Perception, \& Psychophysics, vol. 77, no. 6, pp. 18481853, 2015.

[82] A. M. de C Souza and S. R. dos Santos, "Investigating the distance compression on virtual environments by comparing visualization devices," 2015 XVII Symposium on Virtual and Augmented Reality, pp. 33-41, 2015.

[83] J. W. Kelly, L. A. Cherep, B. Klesel, Z. D. Siegel, and S. George, "Comparison of two methods for improving distance perception in virtual reality," ACM Transactions on Applied Perception, vol. 15, no. 2, 2018.

[84] J. W. Kelly, L. S. Donaldson, L. A. Sjolund, and J. B. Freiberg, "More than just perception-action recalibration: Walking through a virtual environment causes rescaling of perceived space," Attention, Perception, \& Psychophysics, vol. 75, no. 7, pp. 1473-1485, 2013.

[85] Z. D. Siegel and J. W. Kelly, "Walking through a virtual environment improves perceived size within and beyond the walked space," Attention, Perception, E Psychophysics, vol. 79, no. 1, pp. 39-44, 2017.

[86] Z. D. Siegel, J. W. Kelly, and L. A. Cherep, "Rescaling of perceived space transfers across virtual environments," Journal of Experimental Psychology: Human Perception and Performance, vol. 43, no. 10, pp. 1805-1814, 2017

[87] K. A. E. Martens, C. G. Ellard, and Q. J. Almeida, "Does manipulating the speed of visual flow in virtual reality change distance estimation while walking in parkinson's disease?" Experimental Brain Research, vol. 233, no. 3, pp. 787-795, 2015.

[88] Z. Li, E. Sun, C. J. Strawser, A. Spiegel, B. Klein, and F. H. Durgin, "On the anisotropy of perceived ground extents and the interpretation of walked distance as a measure of perception," Journal of Experimental Psychology: Human Perception and Performance, vol. 39, no. 2, pp. 477-493, 2013.

[89] B. Li, R. Zhang, A. Nordman, and S. A. Kuhl, "The effects of minification and display field of view on distance judgments in real and hmd-based environments," SAP 2015: ACM SIGGRAPH Symposium on Applied Perception, pp. 55-58, 2016.

[90] A. Peer and K. Ponto, "Perceptual space warping: Preliminary exploration," 2016 IEEE Virtual Reality Conference, pp. 261-262, 2016.

[91] A. Peer, K. Ponto, and IEEE, "Mitigating incorrect perception of distance in virtual reality through personalized rendering manipulation," 2019 26TH IEEE Conference on Virtual Reality and 3D User Interfaces, pp. 244-250, 2019.

[92] L. Buck, R. Paris, and B. Bodenheimer, "Distance compression in the HTC vive pro: A quick revisitation of resolution," Frontiers in Virtual Reality, vol. 2, 2021.

[93] F. Ding, S. Sepahyar, and S. Kuhl, "Effects of brightness on distance judgments in head mounted displays," in ACM Symposium on Applied Perception 2020. New York, NY, USA: Association for Computing Machinery, 2020.

[94] J. W. Kelly, T. A. Doty, M. Ambourn, and L. A. Cherep, “Distance perception in the oculus quest and oculus quest 2," PsyArXiv, 2022.

[95] K. P. Burnham and D. R. Anderson, "Multimodel inference: un- 
derstanding aic and bic in model selection," Sociological methods $\mathcal{E}$ research, vol. 33, no. 2, pp. 261-304, 2004.

[96] M. R. Symonds and A. Moussalli, "A brief guide to model selection, multimodel inference and model averaging in behavioural ecology using akaike's information criterion," Behavioral Ecology and Sociobiology, vol. 65, no. 1, pp. 13-21, 2011.

[97] K. P. Burnham and D. R. Anderson, Model Selection and Multimodel Inference: A Practical Information-Theoretic Approach. New York: Springer, 2002

[98] J. W. Kelly, J. M. Loomis, and A. C. Beall, "Judgments of exocentric direction in large-scale space," Perception, vol. 33, no. 4, pp. 443454, 2004.

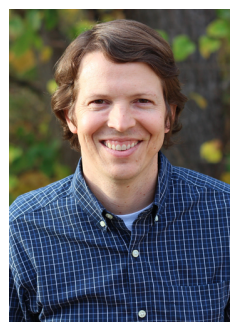

Jonathan Kelly is a professor of Psychology at lowa State University, where he participates in the Human Computer Interaction graduate program. Prior to joining lowa State, he earned his Ph.D. from University of California, Santa Barbara and conducted postdoctoral research at Vanderbilt University. His research interests include virtual reality, navigation, and spatial cognition. 